\title{
Van Buchem Disease: First Case Report from the Indian Subcontinent with an Early Presentation
}

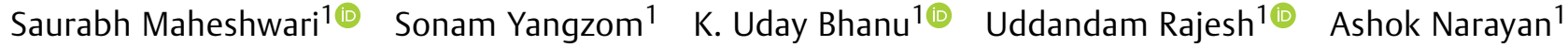 \\ ${ }^{1}$ Department of Radiodiagnosis and Imaging, Armed Forces Medical \\ College, Pune, Maharashtra, India \\ Address for correspondence Saurabh Maheshwari, MD, DNB, \\ Department of Radiodiagnosis, Armed Forces Medical College, Pune \\ 411040, Maharashtra, India (e-mail: saurabhmhshwr@yahoo.co.in).
} J Child Sci 2021;11:e38-e41.

\begin{abstract}
Keywords

- Van Buchem disease

- cranial nerve

- hyperostosis corticalis generalisata

Van Buchem disease is a rare autosomal recessive genetic disorder that causes a compromised inhibitory feedback mechanism resulting in increased bone formation and overgrowth of the skeleton leading to a variety of neurological symptoms. It has been reported in less than 50 patients most of which were in western Europe. We report the first case of this condition from the Indian subcontinent with an early presentation. This patient presented with a global delay in attaining the developmental milestones and progressive reduction in visual acuity and loss of hearing. He had dysmorphic facies, multiple cranial nerve palsies, and severe visual and auditory deficits. Imaging revealed sclerosing bone dysplasia. This case illustrates the clinical and imaging findings of this rare condition.
\end{abstract}

\section{Introduction}

Van Buchem disease (VBD) is characterized by the increased bone formation and overgrowth of the skeleton. It is listed as entry \# 239100 in Online Mendelian Inheritance in Man (OMIM) database. It derives its name from its first documentation by Van Buchem in $1955 .{ }^{1}$ Clinically facial dysmorphism, progressive cranial nerve involvement, and increased intracranial pressure are noted. It most commonly involves the skull and skull base, the mandible, the clavicles, the ribs, and the diaphysis of the tubular bones. We report the case of a 5-year-old boy with classical imaging features of VBD. It is the first case of this condition from the Indian subcontinent. This case is also unique in its early presentation.

\section{Case Report}

A 5-year-old boy was brought to the hospital by caregivers with a global delay in attaining the developmental milestones and progressive reduction in visual acuity and loss of hearing. The symptoms started at the age of 3 years. The child was the product of a nonconsangui-

received

September 17, 2020

accepted after revision

December 15, 2020
DOI https://doi.org/

10.1055/s-0041-1723956

ISSN 2474-5871. neous marriage. There was no family history of similar symptoms.

Clinical examination revealed facial dysmorphism with macrocephaly, flat prominent nasal bridge, hypertelorism, prognathic mandible, and protruding tongue $(-$ Fig. $\mathbf{1})$. The patient had short stature with a global developmental delay. The child had bilateral seventh cranial nerve palsy (HouseBrackmann grade III on the right side and grade IV on the left side) on examination.

The ophthalmologic examination found reduced visual acuity having only light perception bilaterally. The child also had horizontal nystagmus at extremes of gazes with temporal pallor and altered pigmentation at the macula. Visual evoked potential study showed no recordable potentials. Otological examination showed intact tympanic membranes on both sides. Pure tone audiometry revealed severe bilateral mixed conductive and sensorineural hearing loss. The dental examination showed malocclusion, mild decay, and diastema between lower central incisors. The rest of the clinical examination was unremarkable.

Given the suspicion of a syndromic disorder, the patient had a skeletal survey. Posteroanterior and lateral view radiographs

\section{(c) 2021. The Author(s).}

This is an open access article published by Thieme under the terms of the Creative Commons Attribution License, permitting unrestricted use, distribution, and reproduction so long as the original work is properly cited. (https://creativecommons.org/licenses/by/4.0/)

Georg Thieme Verlag KG, Rüdigerstraße 14, 70469 Stuttgart, Germany 


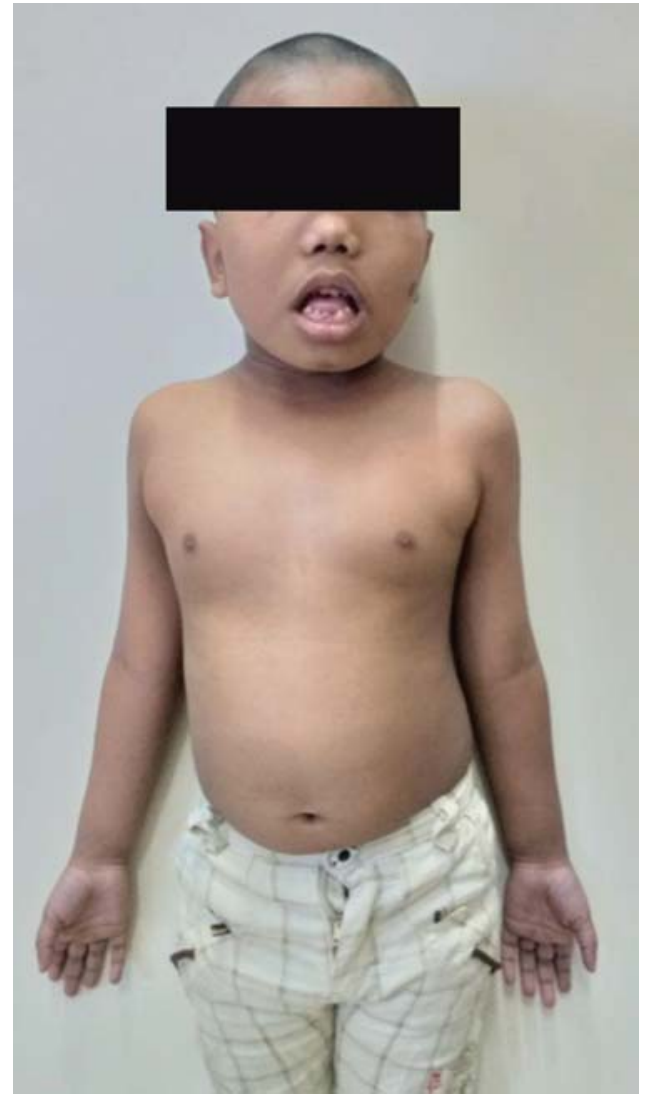

Fig. 1 Frontal photograph of the patient shows facial dysmorphism with a flat nasal bridge, prognathic mandible, and protruding tongue.

of the skull ( - Fig.2) showed generalized thickening of the skull vault, maxilla, and mandible involving both inner and outer tables with partial obliteration of diploic spaces. The skull had a patchy copper-beaten appearance. The maxillary sinus was not visualized. The rest of the skeletal survey was normal.

A corroborative noncontrast computed tomography scan of head was performed which confirmed the findings seen on skull radiograph (-Fig. 3). Characteristic bony excrescences were seen along the inner table of the skull. Also, there was a narrowing of the bilateral optic canals, internal auditory meatuses, and facial nerve canals. Diffuse hyperostosis of the temporal bone was seen to cause severe narrowing of the middle ear cleft. The scan had a Computed Tomography Dose Index of 32.3mGy.

The child had reduced vitamin D3 levels at $9.3 \mathrm{ng} / \mathrm{mL}$ (reference values: $12-20 \mathrm{ng} / \mathrm{ml}$ ) with mildly raised alkaline phosphatase levels at 382 units/liter (reference values for age: 92-309 U/L). The rest of the hematological and biochemical investigations was normal including serum calcium, serum phosphorus, serum parathyroid hormone levels, and urine calcium/creatinine ratio was within the normal range. We could not perform serum osteocalcin level analysis. Genetic analysis could not be done as caregivers did not provide the consent for the same.

The patient is being managed by nonsurgical measures. He has been prescribed hearing aids with mild improvement in the hearing and has been started on glucocorticoids to

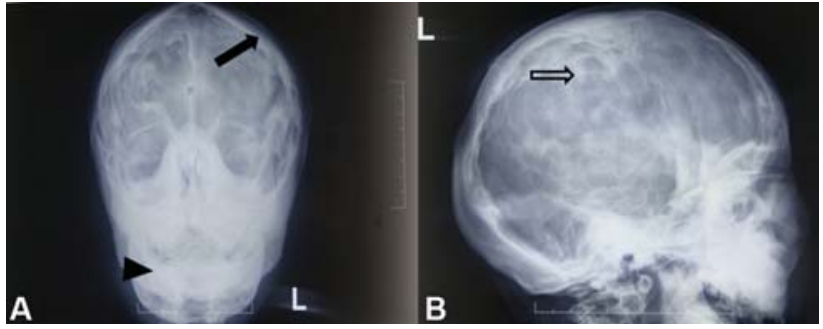

Fig. 2 Radiograph skull posteroanterior (A) and lateral view (B) of the skull shows diffuse calvarial hyperostosis with partial obliteration of diploic spaces (solid black arrow). Similar changes are also seen involving the mandible and maxilla (black arrowhead). The lateral view (B) shows patchy areas showing a cooper-beaten appearance (void black arrow).

suppress further bone formation. His parents have undergone genetic counselling. The child is on regular follow-up.

\section{Discussion}

VBD is an uncommon autosomal recessive hereditary condition with bone dysplasia. ${ }^{2}$ Recessive type of VBD (OMIM 239100) segregates with 17q21.31 locus harboring SOST. There is a homozygous $52-\mathrm{kb}$ deletion downstream of the SOST gene that is thought to decrease gene expression of sclerostin. Sclerostin is an inhibitor of bone formation. The reduction in its expression leads to unrestricted bone

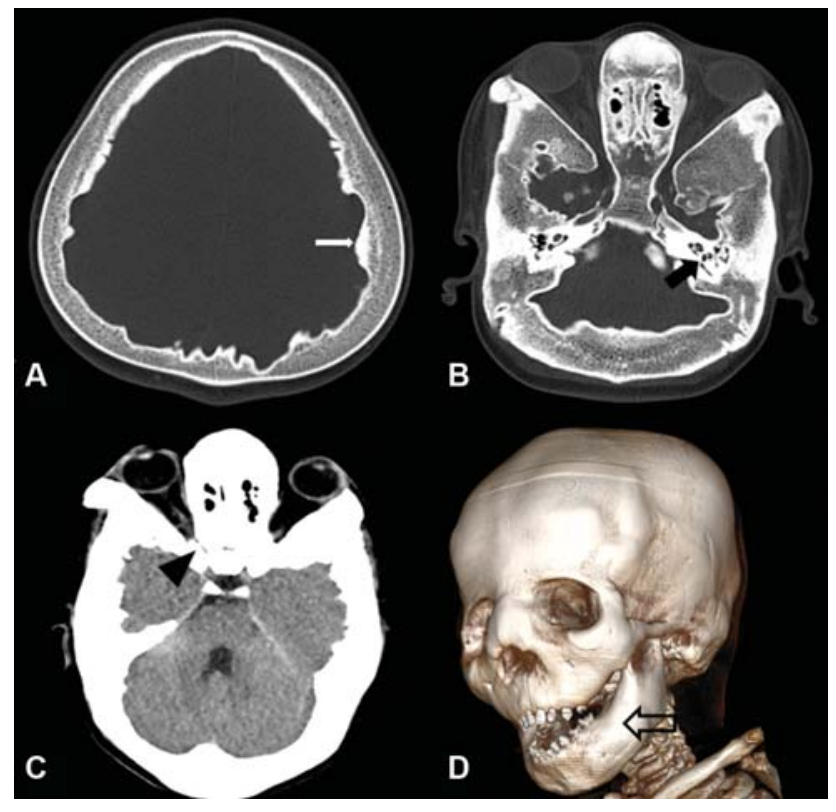

Fig. 3 Computed tomography (CT) images in axial plane in the bone window (A and $\mathbf{B}$ ) show diffuse hyperostosis of calvarium and base of the skull with bony excrescences seen arising from the inner table of the skull (solid white arrow in A). Diffuse hyperostosis involving the temporal bone is seen to cause severe narrowing of the middle ear cleft and encroachment of inner ear structures (solid black arrow). Oblique axial CT reformat image in soft tissue window (C) demonstrates severe narrowing of right optic nerve canal leading to severe compression of the right optic nerve (solid black arrowhead in C). Volume rendered image of the skull (D) shows severe mandibular hyperostosis (void black arrow) in addition to the above findings. 
formation. Autosomal dominantly segregating VBD, type 2 (OMIM 607636) segregates with 11q13.2 locus harboring LRP5.

VBD is extremely rare with less than 35 cases described. It is most prevalent in a small fishing village in the Netherlands. ${ }^{3}$ There are a few isolated case reports from other parts of the world including Germany and Taiwan, but none from the Indian subcontinent.

The disease usually presents with symptoms secondary to cranial bone overgrowth resulting in macrocephaly, visual problems, hearing loss, neurologic pain, and progressive blindness due to optic nerve atrophy. The usual age of onset of cranial nerve palsy is during puberty ${ }^{4}$ with the progression of symptoms throughout life. The most common cranial nerves to be involved are the seventh and eighth cranial nerves. Late neurological complications are raised intracranial pressure and spinal stenosis.

Common imaging findings are massive hyperostosis of the skull and mandible, resulting in increased weight of skull and sclerosis of the diaphysis of the long bones, clavicles, ribs, and pelvis. There is a disruption of bone contours resulting in a rough bone surface. Bone anomalies are symmetrical and apparent in the first decade of life, becoming more prominent with age.

VBD is one of the four conditions grouped as hyperostosis corticalis generalisata with the other three being Sclerosteosis, Truswell-Hansen disease, and Nakamura disease. ${ }^{5}$ VBD is differentiated from these by characteristic features that include stark enlargement of the mandible and small periosteal excrescences seen along the affected bones. Cranial nerve involvement is also a distinguishing feature for VBD. In contrast, Sclerosteosis also shows excessive height, syndactyly, and nail dysplasia. Worth disease and Nakamura disease characteristically do not show cranial nerve palsies. ${ }^{5}$

Other differential diagnoses include Camurati-Engelmann disease (progressive diaphyseal dysplasia) and osteopetrosis. Camurati-Engelmann disease is characterized by the sclerosis and fusiform enlargement of the diaphysis of the long bones. ${ }^{6}$ Osteopetrosis is characterized by generalized bony sclerosis with multiple fractures. However, the degree of calvarial hyperostosis is not as pronounced as $\mathrm{VBD}^{7}$.

Our case demonstrates a few remarkable features. First, this is the only case to be reported from the Indian subcontinent. Second, our patient had onset of cranial nerve palsies with progressive blindness at 3 years of age which is quite uncommon. Only four cases have been described with such an early presentation to date. ${ }^{3,8}$ The finding of mildly raised alkaline phosphatase is consistent with previous studies in VBD. ${ }^{9}$

We acknowledge the lack of genetic testing and serum osteocalcin level measurement in our study. However, we feel that clinical and imaging features are sufficiently specific for VBD in our case. Our case did not show changes in the appendicular skeleton and ribs that may be explained by the young age of the patient. The largest case series on $\mathrm{VBD}^{4}$ describes that cortical thickening and medullary canal widening are two features that are a function of age and appear late in the disease course. The largest case series on pediatric presentation of $\mathrm{VBD}^{3}$ also does not describe appendicular or rib changes in their patients.
The treatment of VBD is symptomatic as no cure is available. Various treatment modalities range from medical therapy in the form of glucocorticoids to surgical interventions in the form of bone-anchored hearing aids, surgical nerve decompression, and decompressive craniectomy. ${ }^{3}$ A detailed discussion of these is beyond the present submission.

\section{Ethics Approval and Consent to Participate}

Ethical approval was waived by the local Ethics Committee of Armed Forces Medical College given the retrospective nature of the study and all the procedures being performed were part of the routine care. The patient reported in this article had signed a written informed consent form to participate in the study and have their data published in a journal article under anonymity. This case report was a reporting of a case in a medical educational center, in which all patients are informed that they may be subjects of scientific experiments and are informed of the ethical codes of conduct. This study was in compliance with the latest version of the Helsinki Declaration.

\section{Consent for Publication}

The patient had written and signed an informed consent note that the findings may be published without any personal detail.

\section{Availability of Data and Material}

All data are available based on a reasonable request.

\section{Authors' Contributions}

All authors contributed to the study conception and design. Material preparation, data collection, and analysis were performed by S.M., A.N. SH, and U.R. The first draft of the manuscript was written by S.Y. and K.U.B. All authors commented on previous versions of the manuscript. All authors read and approved the final manuscript.

All authors have agreed to both to be personally accountable for their contributions and they have ensured that questions related to the accuracy or integrity of any part of this work, even ones in which they were not personally involved, were appropriately investigated, resolved, and the resolution was documented in the literature.

\section{Funding}

None.

\section{Conflict of Interest}

None declared.

\section{Acknowledgments}

We would like to thank Dr. Samaresh Sahu for his support in conducting this study.

\section{References}

1 Van Buchem FS, Hadders HN, Ubbens R. An uncommon familial systemic disease of the skeleton: hyperostosis corticalis generalisata familiaris. Acta Radiol 1955;44(02):109-120 
2 Balemans W, Patel N, Ebeling M, et al. Identification of a $52 \mathrm{~kb}$ deletion downstream of the SOST gene in patients with van Buchem disease. J Med Genet 2002;39(02):91-97

3 van Egmond ME, Dikkers FG, Boot AM, van Lierop AH, Papapoulos SE, Brouwer OF. A rare cause of facial nerve palsy in children: hyperostosis corticalis generalisata (Van Buchem disease). Three new pediatric cases and a literature review. Eur J Paediatr Neurol 2012;16(06):740-743

4 Vanhoenacker FM, Balemans W, Tan GJ, et al. Van Buchem disease: lifetime evolution of radioclinical features. Skeletal Radiol 2003; 32(12):708-718

5 Ihde LL, Forrester DM, Gottsegen CJ, et al. Sclerosing bone dysplasias: review and differentiation from other causes of osteosclerosis. Radiographics 2011;31(07):1865-1882
6 Vanhoenacker FM, Janssens K, Van Hul W, Gershoni-Baruch R, Brik R, De Schepper AM. Camurati-Engelmann disease. Review of radioclinical features. Acta Radiol 2003;44(04):430-434

7 Wengenroth M, Vasvari G, Federspil PA, Mair J, Schneider P, Stippich C. Case 150: Van Buchem disease (hyperostosis corticalis generalisata). Radiology 2009;253(01):272-276

8 Fryns JP, Van den Berghe H. Facial paralysis at the age of 2 months as a first clinical sign of van Buchem disease (endosteal hyperostosis). Eur J Pediatr 1988;147(01):99-100

9 Wergedal JE, Veskovic K, Hellan M, et al. Patients with Van Buchem disease, an osteosclerotic genetic disease, have elevated bone formation markers, higher bone density, and greater derived polar moment of inertia than normal. J Clin Endocrinol Metab 2003;88(12):5778-5783 